\title{
Application of Visual Science Achievement in Artistic Creation
}

\author{
Xin Tao \\ Liaoning He-Medical University \\ Shenyang, China 110163
}

\begin{abstract}
Eyes are the wind of the mind, that's why people have always believed what they see with eyes are real and the most beautiful. Working like a hidden law regulation, it constrains the development of the art to a large extend, because visual science always has different forms during artistic creation, which may be not consistent with visual images. Essentially, visual sense is an embodiment of physical phenomenon, and "light" is the basis for us seeing the boundless universe. Exploring visual theories and applying them in artistic creation are good attempt, and their application in graphic design and interior and exterior design and other fields can give full play to the advantages and characteristics of visual sense. This paper illustrates the relationship between art and science and discusses the visual science from several aspects of artistic creation.
\end{abstract}

keywords-artistic creation; visual science achievement; architectural visualization; science and art

\section{INTRODUCTION}

Visual sense of human beings to exterior world is based on the refraction of "light", which is refracted into the eyes; but sometimes, the eyes may have wrong sense, i.e. illusion in static and dynamic observation, which gives birth to the art. Through long-term observations and conclusions, Da Vinci discovered the principle of perspective, which not only broke through the shallow understanding of imaging principle, but also largely enhanced the aesthetic level of painting. Perspective is a phenomena of physical science; however, if a combination of the perspective and art can be effectively made, it will be a major breakthrough and innovation in art, and it will also introduce new understandings of sculpture, painting and even aesthetic. Therefore, it is very necessary to combine science with art in artistic creation.

\section{RELATIONSHIP BETWEEN SCIENCE AND ART}

\section{A. Similar Representations, Different Essences}

Some people believe science and art are two completely opposite subjects, even without any common features to be described together. Science can be divided into natural science and social science. Natural science consists of mathematics, physics, chemistry, geography, astronomy and biology, while human science covers literature, history,

This paper is one of the general project research results of colleges and universities in Liaoning Province, Project name: Study on Application of Visual Science Achievement in Artistic Creation, Project No.: W2014378. philosophy and other aspects. However, although the art is poor in logical and empirical terms, it can create unconstrained works that do not comply with the law. On the other hand, they have similarities, for example, the geometry knowledge is needed in painting, physical imaging principle is used in photography, and architecture requires the applications of mechanics, material science, esthetics and other subjects. Different scholars have different points of views, without reaching a common answer; but this does not hinder the development of art and science, as non-deliberate combinations imperceptibly build subtle connections. Professor Jiang Xiaoyuan pointed out that no matter the arguments focus on "science and art are interlinked" or "science and art are not interlinked", it will be inevitable to get evidences, which will be hard to obtain no matter for proving either argument; there may be some evidences, but not enough to prove either argument. To sum up in a word, science and art have some relationship in superficial aspects, but they are not interlinked in essence.

\section{B. Binary Opposition View and Common Origin}

Science history reflects the objective law between the development and changes of nature, society and thinking, etc., and it mainly focuses on obtaining objective results through logical deduction and obtaining universal law and knowledge about the external world through complete observations. While art is creations of objects, images, sounds, movement and other different expression forms implying aesthetics by means of imaginations, skills, experience and other subjective factors. Therefore, in theory, people usually distinguish art and science in terms of the subjective and aesthetic aspects, defining them unrelated from two aspects of subjective objectivity and logic aesthetic. Based on such point of view, Carolin A Jones and Peter Galison summed up the Binary Opposition View-intuition and analysis, induction and deduction, visual and logic, random and systematic, independent and cooperative, male and female, and creation and discovery. However, such opposition view was soon challenged and questioned. If according to this view, the research objects of visual science history should be images related to science, excluding art paintings; however, scientists in fact cannot clearly define the boundary between scientific images and art paintings, which means artists and scientists have close cooperation in some respects. In our opinion, both science and art embraced rapid development in the Renaissance period, the historical 
characteristics of which period became indispensable historical background of visual science subject, embodying the pursuit of power of rationality and wisdom. Many artists in the Renaissance period would improve their ability of accurately representing objects by scientific observations and researches; similarly, artistic accomplishments of scientists helped themselves move further in their scientific researches. Just as science and art are closely related to each other, no one can definitely tell which subject is more important.

\section{THE CONCEPT OF VISUAL SCIENCE}

Nowadays, the emerging field of science history research seems to be able to give a deeper level of proof of the similarities between science and art. Since 1980s, with the international academic circles paying more and more attention to visual culture and the circle of science as well as the circle of art deepening the research on scientific image representations in the History of Science, an emerging research field, Visual Science History, gradually appeared from 1990s.

Visual Science History covers a lot of subjects, including science and history of science, art and history of art, history and historiography, and it is an interdisciplinary research of a real sense. With the changing of methods of research topics, it will involve many other academic fields, such as philosophy, art philosophy, cognitive psychology, iconology, semiology, anthropology, linguistics, cultural studies and even religious history, etc. In view of historical origin, Visual Science History links the science and art in the aspect of visual art.

\section{RELATIONSHIP BETWEEN ART AND VisUAL SCIENCE}

\section{A. Photographic Art and Visual Science}

Dating back to 1839 and after a long development history of more than 170 years during which photography experienced many setbacks and improvements, commercial photography was gradually divided from photography. Now, commercial photography art has influenced our life in many aspects. Commercial photography attracts people's eyes by means of snapshotting business process, and its peculiar visual effects always leave people deep impressions, achieving the targets of promoting consumption and raising awareness. Commercial photographers create wonderful works with their unconstrained imagination and sharp insights, leaving the audience a visual feast.

\section{1) Visual forms of commercial photography}

Commercial photography is a branch of photography, and the major difference is its commercial properties. Different from other branches of photography, commercial photography not only captures instant impressions, but also targets at completely presenting commodity information, so that consumers can feel the content of photography. For different consumers, commercial photography will present commodity characteristics in different perspectives, which makes commercial photography more and more popular in real life. Photographers need to first find out the core information of commodities, grasping commodity subjects, so that they can design satisfied prototype with their photographic equipment, then create satisfied commercial photos with their techniques. In recent years, people put much effort into commercial photography, and visual form not only focuses on "visual culture" research, but also extends to the study on "visuality" culture.

Visual form which is put forward in commercial photography has a unique feature: image composition is conducted on the basis of grasping visual elements. In the nature, there are various kinds of lines, which are dominant compositional elements, and these lines support the frame of structure image, extending from two-dimensional plane to three-dimensional space, so as to express deep meaning in different visual angles and viewing distance. The center of commercial photography composition is the necessary way to reach visual sense of form, based on which clear and complete expression of images can be achieved, deepening the presence of commodities and enabling the public with more perfect visual enjoyment. In the process of commercial photography, collisions and combinations between visual elements extend visual sense from one form to another, making visual images more smooth and natural; together with the feature of concise language and integration with the subjects, the effect that all elements to be presented are included in the image can be reached. Commercial photography works highlight image subjects by ignoring some fragments, strengthening inherent expressiveness.

Visual images refer to the combination of all social forms which can be perceived in the nature, including "shape" and "state" of objective things. "Shape" is the form of an object, referring to an expression form; "state" refers to expressed internal development of an object within certain range, and the range relates to all spatial relations and visual relations. "Form and state" highlight the state of existence and dynamic state, and visual form and state combine images with culture, containing multiple meanings like thinking, culture, aesthetic and among others.

Modern photography is different from photography, as it highlights psychological perception of "visual sense" in visual image, equivalent to a psychological perception of the image. Perception is made by visual nerve cells, then information is transmitted to the brain which process the information and produce a visual image. Visual sense can be either a simple way of feeling, or an accumulative feeling based on experience; different shapes and colors of objects can arise different psychological feelings, that's why we say "colors have temperatures and emotions". "Photism" refers to the senses of taste, touch, pain and smell when observing objects in different light environment, and the textures, shapes and states of objects can bring us different feelings. These wonderful synesthesia are brought by life experience as well as cultural accumulation, and colorful synesthesia make our subjectivity diverse and beautiful.

\section{2) Visual language in commercial photography}

In order to understand the business language in the context of art, we need to study the creative thinking of visual language from different perspectives, so as to extend the denotation and connotation of visual language. The 
introduction of new concepts like structuralism, Bauhaus and surrealism broaden the development space of commercial photography. Unique characteristics of visual language can be found through analysis from different perspectives and comparisons with other art categories.

All forms of artistic expression have their own language systems, and due to short development history, the language of commercial photography still needs planning; language is a kind of scientific system and its develop follows specific rules; therefore, the study on system language of commercial photography needs certain scientific methods; the methods of comparison and borrowing are adopted for analyzing the evolution of visual language system of commercial photography, including the perspectives of color, lighting, shooting techniques and lens features. Focal length lens include standard lens, zoom lens, telephoto lens, wide-angle lens and macro lens, and each lens has its own unique features, making itself suitable for shooting different objects and themes. Standard lens has the closest visual effect like human eyes, making shooting images close to their natural states; however, just as close to natural state, the visual impact is not strong enough and special effects are not obvious. Wide-angle lens can enlarge view angle, broaden the depth of field and enhance perspective sense even in close shoot, which attract consumers. Telephoto lenses has the features of small view angle and large imaging, making it suitable for long distance shooting, enabling photographers capturing natural pictures at long distance. Macro lens is suitable for tiny objects, such as insects and flowers. Commercial photography is like the communications between people with different accents and personality, with some being straight-talking and others euphemistic; different languages form different unique images, which is the scientific feature of visual art.

\section{B. Three-dimensional Painting Art and Visual Science}

Cubism splits, flips and reconstruct objects, and expresses forms and emotions in architectural photography by adopting the dimensions representing space and time in two-dimensional space. The application of innovations of cubism photography is a kind of new try, breaking some principles and methods of photography and creating more and more works with unique features.

There are three kinds of cubist painting expressionsbroken objects, planarity and blanking. Traditional composition method is based on the integrity of the image objects, while cubism paintings break this form of reproduction, and it splits up the object and restore it into 2D geometric figures, then reconstruct the figures into a broken object which is called the broken object form; cubism paintings use geometric shapes to organize objects, and these geometric elements themselves do not have any 3D illusion, but after squeezing the features of different lateral sides into one plane which seems to be a flat 2D geometric shape, it will include a lot of subject information; cubism adopts subject blanking or depicts multiple subjects at the same time to eliminate vision center, breaking the sense of wholeness of images. These incredible peculiarities present unique style through appropriate applications, especially in architecture field.

Cubism normally takes human beings as the major subject, while architectural photography takes modernism architecture as the major subject; buildings have very few component elements, which is low in identification and is not very suitable for Cubism, however, if the ceiling part, main structure and bottom part are split and reconstructed, a mellow effect can be reached, avoiding over strong visual impact, so that the negative effect on the subject can be avoided. Architecture is three-dimensional, but if the threedimensional vision is converted plane, and combined with particular shooting techniques and post processing, a Cubism style with plane 2D features can be achieved. In order to weaken the three-dimensional sense of space, the shooting should be carried out in light sources of diffused reflection, such as in cloudy day, the shadow boundaries of buildings will be blurred, so that required effect can be achieved. It can be concluded that visual effect can present $3 \mathrm{D}$ objects in images with plane feelings, which can only be achieved by visual science; therefore, science is indispensable.

\section{Architecture Art and Visual Science}

\section{1) Architecture visual modelling design}

Architecture visual modelling design is an important aspect of architectural design; as a branch of design, architecture visual modelling design includes structural design, color culture design, material design and many other aspects; in the process of practice, architecture visual modelling design focuses on the changes of subtle aspects like dots, lines and planes, and large overall changes are achieved through such changes of subtle aspects. Concrete is the essential material of building construction, however it also has excellent artistic expression, as its rugged and pristine nature can express the "rough" effect of buildings, and deliberately roughness treatment can be conducted during construction to better express the rhythm of buildings. In the visual art, "dot" is the smallest element; it has centripetal force and the most inaccurate shape; it is normally used to represent positions, without length or width; abstracting some parts into dots can help achieve the overall effect of the image. Creative inspirations on visual modeling elements in architecture constantly spring up, creating works following material properties.

\section{2) Super visual form}

The research scope of super visual form analogy design includes the macro super visual form, microscopic visual form and other super visual forms. Using some modern technical equipment, such as astronomical telescope, spacecraft, optical microscope and electron microscope, etc., can clearly observe all kinds of super visual forms; advanced science and the application of super visual form in architecture design are inseparable. Today, with the rapid development of science and technology, especially the development on the synthesis and application of new materials, it is no longer a problem of using nanotechnology materials to meet the needs of any modelling in architectural design, which makes it possible for the development of super visual form and for its gradual improvement. 


\section{CONCLUSION}

To sum up, science and art, to some extent, are complementary and indivisible, and they are combined to form a cross subject which integrates artistic sensibility and scientific preciseness, and art design and creation will open up a new development direction, even creating new schools of development in the future. Today, artistic creations in photography, architecture and other fields have already obtained a lot of achievements and innovations; various billboards with unique design and all kinds of buildings show the potential value of artistic creation in scientific research. Visual imaging is anyhow inseparable with the imaging theory of light in physics; in photography, various lighting technologies are adopted to create images with various peculiar illusions, and scientific materials are used to build artwork with various unusual shapes, creating vivid artwork with depth of field effect. If blindly dividing science into rationality category and dividing art into sensibility category, breakthrough and innovations will be difficult to achieve; exploring new modes to enable the coexistence of science and art will be bound to trigger a big change in both fields. Visual cognition is the most direction cognition of human beings to external world, and it is controllable and brings people more reality; however, visual arts are often illusory or use illusions to achieve magical effects; under the guidance of science, artistic creation will expand more perspectives to observe the world, bringing about a new development direction for artistic creation. Visual science principles are always found in aesthetics, and some phenomena and laws all have scientific reasons in behind; new inventions and creations also are often unexpected results of unintentional creations; art will widen our horizon, protecting us from being limited in vicious circles, and when we stand in the outreach of the world, all problems will be readily solved.

\section{REFERENCES}

[1] User manual and technical conditions of finite element analysis and software design (wall metamodel) of multi-storey and high-rise building structure space. [j]. PKPM CAD [j]. CAD Engineering Department of China Academy of Building Research, Oct. 2008.

[2] Hu Wangshe, Dai Lei, Lin Tao. Interior Modeling Element-Design and Creativity of the Ground. [j]. Chongqing Architecture, Sep. 2009.

[3] Translated by Li Zhicong et al, Textbook Volume II of the New York Institute of Photography. [j]. Edited by New York Institute of Photography, China Photographic Publishing House, 2000.5.

[4] Rudolf Arnheim. Translated by Teng Shou. Visual thinking: aesthetic intuition psychology. [j]. Chengdu: Sichuan Publishing Group, Sichuan People's Publishing House, 1998.

[5] Heinz.Paetzold. Translated by Gao Jianping. Symbol, Culture, City: Five topics of cultural criticism philosophy. [j]. Sichuan Publishing Group, Sichuan People's Publishing House, 2008.

[6] Kent C. Bloomer, Charles W. Moore. Translated by Cheng Chaohui. Body, Memory and Architecture: basic principles and rationale of architectural design. [j]. China Academy of Art Publishing House, 2008.

[7] Shen Yandong, Liu Wenqiao. Exploration on Contemporary Commercial Photography Teaching-- Combination of commercial photography teaching and graphics creative thinking. Art and Literature for the Masses, 2012. (12).
[8] Wang Yue. Importance and Expressions of Innovation in Commercial Photography. [j]. Journal of Kunming Metallurgy College, 2012, (28): 68-70.

[9] Robert Venturi. Translated by Zhou Bu. Complexity and Contradiction in Architecture. [j]. China Waterpower Press, 2006.

[10] TaroIgarashi. Translated by Liu Feng, Liu Xiaojin. 16 Chapters about Modern Architecture: Space, Time and the World. [j]. Jiangsu People's Publishing House, 2012.

[11] WenLiChun. Introduction to Photography Teaching Reform of Computer Advertising Design and Production in Secondary Vocational School. [j]. Science\&Technology Information. 2012, (21).

[12] Liu Yanyan. Study on the Application and Communication Effect of Human Body in Advertising Photography. [j]. Zhejiang: Zhejiang Sci-Tech University, 2012. 